University of New Mexico

UNM Digital Repository

Mathematics and Statistics Faculty and Staff

Publications

Academic Department Resources

2010

Fusion of imprecise qualitative information

Florentin Smarandache

Xinde Li

Xianzhong Dai

Jean Dezert

Follow this and additional works at: https://digitalrepository.unm.edu/math_fsp

Part of the Automotive Engineering Commons, Aviation Commons, Mathematics Commons, Navigation, Guidance, Control and Dynamics Commons, Other Aerospace Engineering Commons, Other Operations Research, Systems Engineering and Industrial Engineering Commons, and the Systems Engineering Commons 


\title{
Fusion of imprecise qualitative information
}

\author{
Xinde Li • Xianzhong Dai · Jean Dezert • \\ Florentin Smarandache
}

Published online: 21 February 2009

(C) Springer Science+Business Media, LLC 2009

\begin{abstract}
In this paper, we present a new 2-tuple linguistic representation model, i.e. Distribution Function Model (DFM), for combining imprecise qualitative information using fusion rules drawn from Dezert-Smarandache Theory (DSmT) framework. Such new approach allows to preserve the precision and efficiency of the combination of linguistic information in the case of either equidistant or unbalanced label model. Some basic operators on imprecise 2-tuple labels are presented together with their extensions for imprecise 2-tuple labels. We also give simple examples to show how precise and imprecise qualitative information can be combined for reasoning under uncertainty. It is concluded that DSmT can deal efficiently with both precise and imprecise quantitative and qualitative beliefs, which extends the scope of this theory.
\end{abstract}

Keywords Information fusion - Qualitative reasoning under uncertainty $\cdot D S m T \cdot$ Imprecise belief structures . 2-Tuple linguistic label

\section{Li $(\varangle) \cdot$ X. Dai}

Key Laboratory of Measurement and Control of CSE (School of Automation, Southeast University), Ministry of Education,

Nanjing 210096, China

e-mail:xindeli@seu.edu.cn

\section{J. Dezert}

ONERA (The French Aerospace Lab.), 29 Avenue de la Division Leclerc, 92320 Châtillon, France

e-mail: jean.dezert@onera.fr

F. Smarandache

Chair of Math. \& Sciences Dept., University of New Mexico, 200

College Road, Gallup, NM 87301, USA

e-mail: smarand@unm.edu

\section{Introduction}

Qualitative methods for reasoning under uncertainty have gained more and more attentions by Information Fusion community, especially by the researchers and system designers working in the development of modern multi-source systems for information retrieval, fusion and management in defense, in robotics and so on. This is because traditional methods based only on quantitative representation and analysis are not able to adequately satisfy the need of the development of science and technology that integrate at higher fusion levels human beliefs and reports in complex systems. Therefore qualitative knowledge representation and analysis becomes more and more important and necessary in next generations of decision-making support systems. In 1954, Polya was one of the pioneers to characterize formally the qualitative human reports [19]. Then Zadeh [31-35] made important contributions in this field in proposing a fuzzy linguistic approach to model and to combine qualitative/vague information expressed in natural language. However, since the combination process highly depends on the fuzzy operators chosen, a possible issue has been pointed out by Yager in [30]. In 1994, Wellman developed Qualitative Probabilistic Networks (QPN) based on a Qualitative Probability Language, which relaxed precision in representation and reasoning within the probabilistic framework [29]. Subrahmanian introduced the annotated logics, which was a powerful formalism for classical (i.e. consistent), as well as paraconsistent reasoning in artificial intelligence [15, 27]. QPN and Annotated Logics belong actually to the family of imprecise probability [28] and probability bounds analysis (PBA) approaches [6]. Parsons proposed a Qualitative Evidence Theory (QET) with new interesting qualitative reasoning techniques but his QET unfortunately cannot deal efficiently 
with complex problems of qualitative information fusion encountered in real world [16-18]. Dubois and Prade proposed a Qualitative Possibility Theory (QPT) in Decision Analysis (DA) for the representation and the aggregation of preferences. QPT was driven by the principle of minimal specificity [4]. They use refined linguistic quantifiers to represent either the possibility distributions which encode a piece of imprecise knowledge about a situation, or to represent the qualitative belief masses over the elements in $2^{\Theta}$. However, the combination process might produce approximate results because of the finite probabilistic scale of the label set [5]. Hájek et al. in [9] proposed a Qualitative Fuzzy Possibilistic Logic (QFPL) which was used to deal with both uncertainty (possibility) and vagueness (fuzziness). QFPL is different from our qualitative reasoning in DSmT or DST frameworks, though the propositional variables were mapped to a set of values i.e. $\{0,1 / n, 2 / n, \ldots, 1\}$ similar to 1-tuple linguistic model, since it built modality-free formulas from propositional variables using connectives, i.e. $\wedge, \vee, \rightarrow, \neg$.

The goal of this paper is to propose a mathematical model of imprecise qualitative belief structures for solving fusion problems for decision-making support. Our main concern is to deal efficiently with (potentially highly) conflicting imprecise and uncertain human-based sources of information, since most of modern (and future) systems for decisionmaking support in security and surveillance, in threat assessment, in defense, etc., require the integration of human observers/soldiers and/or expert reports in the loop. Our purpose is not to try compare our approach with all aforementioned theoretical attempts, but only with the few main fusion rules used in the theories of belief functions which fit with the models and operators proposed in this work in order to show what and how the results can be obtained with such new approach. This work pursues the efforts made by our predecessors for a search of robust and efficient ways for dealing with conflicting qualitative sources of information.

Some research works on quantitative imprecise (quantitative) belief structures have been done at the end of nineties by Denœux who proposed a representation model in DST framework for dealing with imprecise belief and plausibility functions, imprecise pignistic probabilities together with the extension of Dempster's rule [1] for combining imprecise belief masses. Within the DSmT framework, Dezert and Smarandache further proposed new interval-valued beliefs operators and generalized $D S m$ combination rules from precise belief structures fusion to imprecise/sub-unitary intervals fusion, and more generally, to any set of sub-unitary intervals fusion [21]. In [13], Li proposed a revised version of imprecise division operator and the Min and Max operators for imprecise belief structures, which can be applied to fuzzy-extended reasoning combination rules. Since all the extensions of belief structures proposed so far in the literature concern only imprecise quantitative belief structures, we introduce here for the first time a representation for imprecise qualitative belief structures. In this paper we present Distribution Function Model (DFM), which offers a less computational complexity by working with a finite reduced/coarse granularity set of linguistic labels [3, 23, 25 ] and a simpler way to deal with unbalanced labels than Herrera-Martínez' model [10] and other [7, 8]. We also introduce new operators based on it for combining imprecise qualitative belief masses, in order to solve fusion problems for decision-making support.

This paper is organized as follows: In Sect. 2, we remind briefly the basis of DSmT. In Sect. 3, we present different linguistic models for qualitative beliefs with the main operators on 2-tuples labels. In Sect. 4, we present the fusion rules for precise and imprecise qualitative beliefs in DSmT framework. In Sect. 5, we provide examples to show how these operators work for combining 2-Tuple qualitative beliefs. Concluding remarks are then given in Sect. 6.

\section{$2 \mathrm{DSmT}$ for the fusion of beliefs}

\subsection{Basic belief mass (bba)}

In Dempster-Shafer Theory (DST) framework [20], one considers a frame of discernment $\Theta=\left\{\theta_{1}, \ldots, \theta_{n}\right\}$ as a finite set of $n$ exclusive and exhaustive elements (i.e. Shafer's model denoted $\mathcal{M}^{0}(\Theta)$ ). The power set of $\Theta$ is the set of all subsets of $\Theta$. The cardinality of a power set of a set of cardinality $|\Theta|=n$ is $2^{n}$. The power set of $\Theta$ is denoted $2^{\Theta}$. For example, if $\Theta=\left\{\theta_{1}, \theta_{2}\right\}$, then $2^{\Theta}=\left\{\emptyset, \theta_{1}, \theta_{2}, \theta_{1} \cup \theta_{2}\right\}$. In Dezert-Smarandache Theory (DSmT) framework [21, 23], one considers $\Theta=\left\{\theta_{1}, \ldots, \theta_{n}\right\}$ as a finite set of $n$ exhaustive elements only (i.e. free $D S m$-model denoted $\mathcal{M}^{f}(\Theta)$ ). Eventually some integrity constraints can be introduced in this free model depending on the nature of problem we have to cope with. The hyper-power set of $\Theta$ (i.e. the free Dedekind's lattice) denoted $D^{\Theta}$ [21] is defined as:

1. $\emptyset, \theta_{1}, \ldots, \theta_{n} \in D^{\Theta}$.

2. If $A, B \in D^{\Theta}$, then $A \cap B$ and $A \cup B$ belong to $D^{\Theta}$.

3. No other elements belong to $D^{\Theta}$, except those obtained by using rules 1 or 2 .

If $|\Theta|=n$, then $\left|D^{\Theta}\right| \leq 2^{2^{n}}$. Since for any finite set $\Theta$, $\left|D^{\Theta}\right| \geq\left|2^{\Theta}\right|$, we call $D^{\Theta}$ the hyper-power set of $\Theta$. For example, if $\Theta=\left\{\theta_{1}, \theta_{2}\right\}$, then $D^{\Theta}=\left\{\emptyset, \theta_{1} \cap \theta_{2}, \theta_{1}, \theta_{2}, \theta_{1} \cup\right.$ $\left.\theta_{2}\right\}$. The free DSm model $\mathcal{M}^{f}(\Theta)$ corresponding to $D^{\Theta}$ allows to work with vague concepts which exhibit a continuous and relative intrinsic nature. Such concepts cannot be precisely refined in an absolute interpretation because of the unreachable universal truth. The main differences between DST and DSmT frameworks are (i) the model on which one works with, (ii) the choice of the combination rule and conditioning rules [21, 23], and (iii) aside working with nu- 
merical/quantitative beliefs DSmT allows to compute directly with words (more exactly to combine qualitative belief masses as we will show in the sequel). Here we use the generic notation $G^{\Theta}$ for denoting either $D^{\Theta}$ (when working in DSmT with free DSm model) or $2^{\Theta}$ (when working in DST with Shafer's model) or any other subset of $D^{\Theta}$ (when working with a DSm hybrid model).

From any finite discrete frame $\Theta$, we define a quantitative basic belief assignment (bba) as a mapping $m():. G^{\Theta} \rightarrow$ $[0,1]$ associated to a given body of evidence $\mathcal{B}$ which satisfies

$m(\emptyset)=0 \quad$ and $\quad \sum_{A \in G^{\Theta}} m(A)=1$

where $G^{\Theta}$ is the generic notation for the hyper-power set taking into account all integrity constraints (if any) of the model. For example, if one considers a free- $D S m$ model for $\Theta$ then $G^{\Theta}=D^{\Theta}$. If Shafer's model is used instead then $G^{\Theta}=2^{\Theta}$ (the classical power-set).

\subsection{Fusion of quantitative beliefs}

When the free $D S m$ model $\mathcal{M}^{f}(\Theta)$ holds, the pure conjunctive consensus, called $D S m$ classic rule $(D S m C)$, is performed on $G^{\Theta}=D^{\Theta}$. DSmC of two independent ${ }^{1}$ sources associated with bba's $m_{1}($.$) and m_{2}($.$) is thus given by$ $m_{D S m C}(\emptyset)=0$ and $\forall X \in D^{\Theta}$ by [21]:

$m_{D S m C}(X)=\sum_{\substack{X_{1}, X_{2} \in D^{\Theta} \\ X_{1} \cap X_{2}=X}} m_{1}\left(X_{1}\right) m_{2}\left(X_{2}\right)$

$D^{\Theta}$ being closed under $\cup$ and $\cap$ operators, $D S m C$ guarantees that $m($.$) is a proper bba.$

When Shafer's model holds, instead of distributing the total conflicting mass onto elements of $2^{\Theta}$ proportionally with respect to their masses resulted after applying the conjunctive rule as within Demspter's rule $(D S)$ through the normalization step [20], or transferring the partial conflicts onto partial uncertainties as within $D S m H$ rule [21], we propose to use the Proportional Conflict Redistribution rule no.5 (PCR5) [22, 23] which transfers the partial conflicting masses proportionally to non-empty sets involved in the model according to all integrity constraints. PCR5 rule works for any degree of conflict in $[0,1]$, for any models (Shafer's model, free $D S m$ model or any hybrid $D S m$ model) and both in DST and DSmT frameworks for static or dynamical fusion problems. The PCR5 rule for two sources is

\footnotetext{
${ }^{1}$ While independence is a difficult concept to define in all theories managing epistemic uncertainty, we consider that two sources of evidence are independent (i.e. distinct and noninteracting) if each leaves one totally ignorant about the particular value the other will take.
}

defined by: $m_{P C R 5}(\emptyset)=0$ and $\forall X \in G^{\Theta} \backslash\{\emptyset\}$

$$
\begin{aligned}
& m_{P C R 5}(X) \\
& =m_{12}(X) \\
& \quad+\sum_{\substack{Y \in G^{\Theta} \backslash\{X\} \\
X \cap Y=\emptyset}}\left[\frac{m_{1}(X)^{2} m_{2}(Y)}{m_{1}(X)+m_{2}(Y)}+\frac{m_{2}(X)^{2} m_{1}(Y)}{m_{2}(X)+m_{1}(Y)}\right]
\end{aligned}
$$

where each element $X$, and $Y$, is in the disjunctive normal form. $m_{12}(X)$ corresponds to the conjunctive consensus on $X$ between the two sources. All denominators are different from zero. If a denominator is zero, that fraction is discarded. No matter how big or small is the conflicting mass, PCR5 mathematically does a better redistribution of the conflicting mass than Dempster's rule and other rules since PCR5 goes backwards on the tracks of the conjunctive rule and redistributes the partial conflicting masses only to the sets involved in the conflict and proportionally to their masses put in the conflict, considering the conjunctive normal form of the partial conflict. PCR5 is quasi-associative and preserves the neutral impact of the vacuous belief assignment. General PCR5 fusion formula and improvement for the combination of $k \geq 2$ sources of evidence can be found in [23] with many detailed examples.

\section{Linguistic models of qualitative beliefs}

\subsection{The 1-tuple linguistic model}

In order to compute qualitative belief assignments expressed by pure linguistic labels (i.e. 1-tuple linguistic representation model) over $G^{\Theta}$, Smarandache and Dezert have defined in [23] a qualitative basic belief assignment $q_{1} m($.) as a mapping function from $G^{\Theta}$ into a set of linguistic labels $L=\left\{L_{0}, \tilde{L}, L_{n+1}\right\}$ where $\tilde{L}=\left\{L_{1}, \ldots, L_{n}\right\}$ is a finite set of linguistic labels and where $n \geq 2$ is an integer. For example, $L_{1}$ can take the linguistic value "poor", $L_{2}$ the linguistic value "good", etc. $\tilde{L}$ is endowed with a total order relationship $\prec$, so that $L_{1} \prec L_{2} \prec \cdots \prec L_{n}$, where $\prec$ means inferior to, or less (in quality) than, or smaller than, etc. To work on a true closed linguistic set $L$ under linguistic addition and multiplication operators, Smarandache and Dezert extended naturally $\tilde{L}$ with two extreme values $L_{0}=L_{\min }$ and $L_{n+1}=L_{\max }$, where $L_{0}$ corresponds to the minimal qualitative value and $L_{n+1}$ corresponds to the maximal qualitative value, in such a way that $L_{0} \prec L_{1} \prec$ $L_{2} \prec \cdots \prec L_{n} \prec L_{n+1}$. In the sequel $L_{i} \in L$ are assumed linguistically equidistant labels such that we can make an isomorphism $\phi_{L}$ between $L=\left\{L_{0}, L_{1}, L_{2}, \ldots, L_{n}, L_{n+1}\right\}$ and $\{0,1 /(n+1), 2 /(n+1), \ldots, n /(n+1), 1\}$, defined as $\phi_{L}\left(L_{i}\right)=i /(n+1)$ for all $i=0,1,2, \ldots, n, n+1$. 
From the extension of the isomorphism between the set of linguistic equidistant labels and a set of numbers in the interval $[0,1]$, one can built exact operators on linguistic labels which makes possible the extension of all quantitative fusion rules into their qualitative counterparts [14]. We briefly remind the basic qualitative operators ${ }^{2}$ (or $q$-operators for short) on (1-tuple) linguistic labels:

- $q$-addition:

$$
L_{i}+L_{j}= \begin{cases}L_{i+j} & \text { if } i+j<n+1 \\ L_{n+1}=L_{\max } & \text { if } i+j \geq n+1\end{cases}
$$

The $q$-addition is an extension of the addition operator on equidistant labels which is given by $L_{i}+L_{j}=\frac{i}{n+1}+$ $\frac{j}{n+1}=\frac{i+j}{n+1}=L_{i+j}$.

- $q$-subtraction:

$$
L_{i}-L_{j}= \begin{cases}L_{i-j} & \text { if } i \geq j \\ -L_{j-i} & \text { if } i<j\end{cases}
$$

where $-L=\left\{-L_{1},-L_{2}, \ldots,-L_{n},-L_{n+1}\right\}$. The $q$ subtraction is justified since when $i \geq j$, one has with equidistant labels $L_{i}-L_{j}=\frac{i}{n+1}-\frac{j}{n+1}=\frac{i-j}{n+1}$.

- $q$-multiplication ${ }^{3}$ :

$$
L_{i} \cdot L_{j}=L_{[(i \cdot j) /(n+1)]},
$$

where $[x]$ means the closest integer ${ }^{4}$ to $x$ (with $[n+0.5]=n+1, \forall n \in \mathbb{N})$. This operator is justified by the approximation of the product of equidistant labels given by $L_{i} \cdot L_{j}=\frac{i}{n+1} \cdot \frac{j}{n+1}=\frac{(i \cdot j) /(n+1)}{n+1}$. A simpler approximation of the multiplication, but less accurate (as proposed in [23]) is thus

$$
L_{i} \times L_{j}=L_{\min \{i, j\}}
$$

- Scalar multiplication of a linguistic label: Let $a$ be a real number. The multiplication of a linguistic label by a scalar is defined by:

$$
a \cdot L_{i}=\frac{a \cdot i}{n+1} \approx \begin{cases}L_{[a \cdot i]} & \text { if }[a \cdot i] \geq 0 \\ L_{-[a \cdot i]} & \text { otherwise }\end{cases}
$$

\footnotetext{
${ }^{2}$ more $q$-operators can be found in [3].

${ }^{3}$ The $q$-multiplication of two linguistic labels defined here can be extended directly to the multiplication of $n>2$ linguistic labels. For example the product of three linguistic label will be defined as $L_{i} \cdot L_{j} \cdot L_{k}=L_{[(i \cdot j \cdot k) /(n+1)(n+1)]}$, etc.

${ }^{4}$ When working with labels, no matter how many operations we have, the best (most accurate) result is obtained if we do only one approximation, and that one should be just at the very end.
}

- Division of linguistic labels:

(a) $q$-division as an internal operator: Let $j \neq 0$, then

$$
L_{i} / L_{j}= \begin{cases}L_{[(i / j) \cdot(n+1)]} & \text { if }[(i / j) \cdot(n+1)]<n+1 \\ L_{n+1} & \text { otherwise }\end{cases}
$$

The first equality in (9) is well justified because with equidistant labels, one gets: $L_{i} / L_{j}=\frac{i /(n+1)}{j /(n+1)}=$ $\frac{(i / j) \cdot(n+1)}{n+1} \approx L_{[(i / j) \cdot(n+1)]}$.

(b) Division as an external operator: $\square$. Let $j \neq 0$. We define:

$L_{i} \square L_{j}=i / j$

Since for equidistant labels $L_{i} \square L_{j}=(i /(n+1)) /$ $(j /(n+1))=i / j$.

From the $q$-operators we now can easily and directly extend all quantitative fusion rules like $D S m C$ or PCR5 into their qualitative version by replacing classical operators on numbers with linguistic labels defined just above in the formulas (2) or (3). Many detailed examples can be found in $[3,14,22,23]$.

\subsection{The precise 2-tuple linguistic model}

The precise 2-tuple linguistic labels representation allows to take into account some available richer information content (if any), like less good, good enough, very good which is not represented within the 1-tuple linguistic labels representation. Herrera and Martínez in [10] were the first to propose a 2-tuple fuzzy linguistic representation model for computing with words $(\mathrm{CW})$ for offering a tractable method for aggregating linguistic information (i.e. Herrera and Martínez model (HMM) represented by linguistic variables with equidistant labels) through counting indexes of the corresponding linguistic labels. The advantages of the 2-tuple Linguistic representation of symbolic method over methods based on the extension principle in $\mathrm{CW}$ in term of complexity and feasibility have been shown in [10].

For the equidistant labels with uniform distribution, it is not difficult to solve. But for an unbalanced label model (as shown in Fig. 1), how to deal with such kind of labels? Though Herrera and Martínez deals with unbalanced labels with Multi-granular Hierarchical Linguistic Contexts in $[11,12]$, whose approach seems too complex in our opinions. In addition, Jin-Hsien Wang and Jongyun Hao proposed another version of 2-Tuple fuzzy linguistic representation model for computing with words by considering a proportional factor as 2 order component [7, 8], which can be transformed to Herrera-Martínez' 2-Tuple linguistic representation model. Here we propose a more general and simpler representation model, called the Distribution Function 


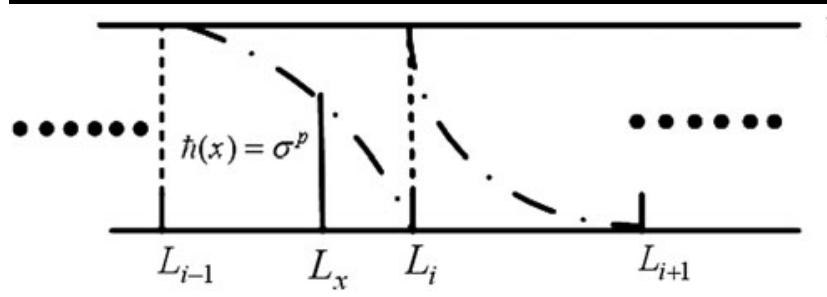

Fig. 1 The 2-Tuple label representation model with unbalanced, or non-uniform distribution

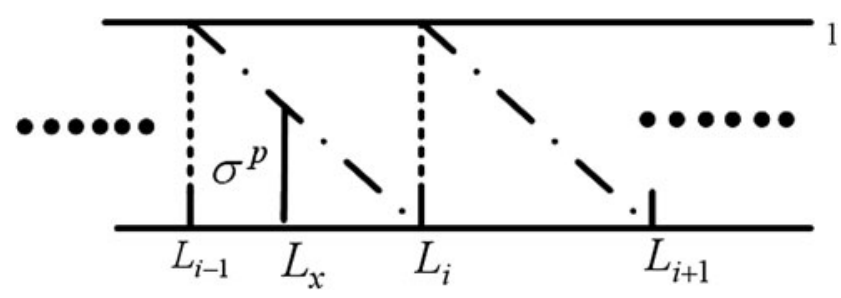

Fig. 2 The 2-Tuple label representation model with the proportional assessment

Model (DFM), which can deal with either equidistant or unbalanced labels.

We assume that there always exist a bundle of distribution functions $\hbar(x)=-(|x|-i+1)^{k}+1,0<k \in R$ between any two labels i.e. $L_{i-1}$ and $L_{i}, i \in[-n, n+1]$, which is continuous and differential (not differential just when $L_{x}=$ $L_{i}, i$ is a integer). Obviously, its 1-order derivative $\frac{d \hbar(.)}{d x}<0$, when $x>0, \frac{d \hbar(.)}{d x}>0$, when $x<0$, because it is an even function, i.e $\hbar(x)=\hbar(-x)$. Therefore, as we know that the inverse function of $\hbar($.$) always exists, i.e. \hbar^{-1}(.) \in[i-1, i]$. So here, we represent this kind of 2-Tuple label model to be $\left(L_{i}, \hbar().\right)$ denoted $q_{2}^{p}$, which is distinct from $\operatorname{HMM}\left(L_{i}, \sigma_{i}^{h}\right)$ denoted $q_{2}^{h}$.

Comparatively to HMM, $i-\hbar^{-1}$ (.) is a remainder from the standard label $L_{i}$. In order to clearly explain this, we give a simple linear distribution function (when $k=1$ ) $\hbar(x)=$ $\sigma^{p}=-|x|+i, x \in[i-1, i]$ shown in Fig. 2.

$\hbar^{-1}()=.i-\sigma^{p}$ is continuous within the interval $[i-1, i], i \in[1, n+1]$, where $\sigma^{p}$ is a proportional factor used as the 2-order component modifier between two neighboring labels, i.e. $\frac{i-x}{i-(i-1)}=\frac{\sigma^{p}}{1}, x=i-\sigma^{p}$. We denote this kind of 2-Tuple label model $\left(L_{i}, \sigma^{p}\right)=L_{x}=L_{i-\sigma^{p}}$, which is a bit similar to Jin-Hsien Wang and Jongyun Hao's work $[9,10]$, but simpler and more generalized than it.

Example Let's consider two labels $L_{i-1}, L_{i}, i \in[1, n+1]$ and let's assume that there is a 2-Tuple label $\left(L_{i}, 0.6\right)$, then, $\left(L_{i}, 0.6\right)=L_{(i-0.6)}$. Of course, if $\left(L_{i}, \sigma^{h}\right)=\left(L_{i}, \sigma^{p}\right)$, there is a relation between them: $i=j, \sigma^{p}=-(n+1) \sigma^{h}$, when $\sigma^{h} \leq 0$, and $j=i+1, \sigma^{p}=1-(n+1) \sigma^{h}$, when $\sigma^{h}>0$, where, if $\sigma^{p}=1$, then $\left(L_{i}, 1\right)=L_{i-1}$. If $\sigma^{p}=0$, then $\left(L_{i}, 0\right)=L_{i}$.
3.2.1 Some useful $q_{2}^{p}$ operators

- Comparison operator:

At first, we can define the comparison operator for any two labels $\left(L_{i}, \hbar(i)\right),\left(L_{j}, \hbar(j)\right)$ under the distribution function model.

(1) if $i>j, i, j \in[-(n+1), n+1]$, there is always the relation $\left(L_{i}, \hbar(i)\right) \geq\left(L_{j}, \hbar(j)\right)$.

(2) if $i=j, i, j \in[-(n+1), n+1]$, and $\hbar(i) \leq \hbar(j)$, then $\left(L_{i}, \hbar(i)\right) \geq\left(L_{j}, \hbar(j)\right)$. Otherwise, $\left(L_{i}, \hbar(i)\right)<$ $\left(L_{j}, \hbar(j)\right)$.

(3) if $i<j, i, j \in[-(n+1), n+1]$, then $\left(L_{i}, \hbar(i)\right) \leq$ $\left(L_{j}, \hbar(j)\right)$.

\section{- Negation operator:}

Of course, the Negation operator is also defined here, in order to satisfy the need of the combination operation.

$\operatorname{Neg}\left(\left(L_{i}, \hbar(i)\right)\right)=\left(L_{-i},-\hbar(-i)\right)$

where, $\hbar(-i)=\hbar(i)$, for example, for $\left(L_{i}, \sigma^{p}\right)$, $\operatorname{Neg}\left(L_{i}, \sigma^{p}\right)=L_{-i+\sigma^{p}}$.

- $q_{2}^{p}$-Addition: For any two labels $\left(L_{i}, \hbar(i)\right),\left(L_{j}, \hbar(j)\right)$, one defines

$\left(L_{i}, \hbar(i)\right)+\left(L_{j}, \hbar(j)\right)=L_{\hbar^{-1}(i)+j+\hbar^{-1}(j)}$.

Special case,

$\left(L_{i}, \sigma_{i}^{p}\right)+\left(L_{j}, \sigma_{j}^{p}\right)=L_{i+j-\sigma_{i}^{p}-\sigma_{j}^{p}}$.

- $q_{2}^{p}$-Subtraction: For any two labels $\left(L_{i}, \hbar(i)\right),\left(L_{j}, \hbar(j)\right)$, one defines

$\left(L_{i}, \hbar(i)\right)-\left(L_{j}, \hbar(j)\right)=L_{\hbar^{-1}(i)-\hbar^{-1}(j)}$.

Special case,

$\left(L_{i}, \sigma_{i}^{p}\right)-\left(L_{j}, \sigma_{j}^{p}\right)=L_{i-j+\sigma_{j}^{p}-\sigma_{i}^{p}}$

- $q_{2}^{p}$-Product: For any two labels $\left(L_{i}, \hbar(i)\right),\left(L_{j}, \hbar(j)\right)$, one defines

$\left(L_{i}, \hbar(i)\right) \times\left(L_{j}, \hbar(j)\right)=L_{\frac{\left(\hbar^{-1}(i)\right) \times\left(\hbar^{-1}(j)\right)}{n+1}}$.

Special case,

$\left(L_{i}, \sigma_{i}^{p}\right) \times\left(L_{j}, \sigma_{j}^{p}\right)=L_{\frac{\left(i-\sigma_{i}^{p}\right) \times\left(j-\sigma_{j}^{p}\right)}{n+1}}$

where, the product operators in (16)-(17) can be easily justified according to the product operator in HMM because of their consistency.

- $q_{2}^{p}$-Scalar multiplication: For any label $\left(L_{i}, \hbar(i)\right), i \in$ $n+1$, and a real number $\alpha$, one defines

$\alpha \cdot\left(L_{i}, \hbar(i)\right)=\left(L_{i}, \hbar(i)\right) \times \alpha=L_{\alpha \cdot\left(\hbar^{-1}(i)\right)}$. 
Special case,

$\alpha \cdot\left(L_{i}, \sigma_{i}^{p}\right)=\left(L_{i}, \sigma_{i}^{p}\right) \times \alpha=L_{\alpha\left(i-\sigma_{i}^{p}\right)}$.

- $q_{2}^{p}$-Division: For any two labels $\left(L_{i}, \hbar(i)\right),\left(L_{j}, \hbar(j)\right)$, if $\left(L_{i}, \hbar(i)\right)<\left(L_{j}, \hbar(j)\right)$, then one defines

$\left(L_{i}, \hbar(i)\right) \div\left(L_{j}, \hbar(j)\right)=L_{\frac{\left(\hbar^{-1}(i)\right)}{\left(\hbar^{-1}(j)\right)} \times(n+1)}$.

Special case,

$\left(L_{i}, \sigma_{i}^{p}\right) \div\left(L_{j}, \sigma_{j}^{p}\right)=L_{\frac{\left(i-\sigma_{i}^{p}\right)}{\left(j-\sigma_{j}^{p}\right)} \times(n+1)}$.

All these operators can be also easily justified and of course, we can easily transform all the operators in (12)(21) to their standard style according to 2-Tuple definition in DFM.

\subsection{The imprecise 2-tuple linguistic model}

Since qualitative belief assignment might be imprecise by expert on some occasions, in order to further combine this imprecise qualitative information, we introduce operators on imprecise 2-tuple labels (i.e. addition, subtraction, product and division, etc.). The definition adopted here is the qualitative extension of the one proposed by Denœux' in [1] for reasoning with (quantitative) Interval-valued Belief Structures (IBS).

Definition 1 (IQBS) Let $\bigsqcup_{G^{\Theta}}$ denotes the set of all qualitative belief structures (i.e. precise and imprecise) over $G^{\Theta}$. An imprecise qualitative belief structure (IQBS) is defined as a non-empty subset $\mathbf{m}$ from $\bigsqcup_{G^{\Theta}}$, such that there exist $n$ subsets $F_{1}, \ldots, F_{n}$ over $G^{\Theta}$ and $n$ qualitative intervals $\left[a_{i}, b_{i}\right], 1 \leq i \leq n$ (with $L_{0} \leq a_{i} \leq b_{i} \leq L_{n+1}$ ) such that

$$
\begin{aligned}
\mathbf{m}= & \left\{m \in t_{G^{\Theta}} \mid a_{i} \leq m\left(F_{i}\right) \leq b_{i}, 1 \leq i \leq n,\right. \\
& \text { and } \left.\left.m(A)=\left(L_{0}, 0\right)\right), \forall A \notin\left\{F_{1}, \ldots, F_{n}\right\}\right\}
\end{aligned}
$$

Proposition 1 A necessary and sufficient condition for $\boldsymbol{m}$ to be non-empty is that $\sum_{i=1}^{n} a_{i} \leq L_{n+1}$ and $\sum_{i=1}^{n} b_{i} \geq L_{n+1}$ (by extension of Denæeux' proposition [1]).

In order to combine imprecise qualitative belief structures, we use the operations on sets proposed by Dezert and Smarandache in [2].

\subsubsection{Addition of imprecise 2-tuple labels}

The addition operator is very important in most of combination rules for fusing information in most of belief functions theories (in DST framework, in Smets' Transferable Belief Model (TBM) [26] as well as in DSmT framework). The addition operator for imprecise 2-tuple labels (since every imprecise mass of belief is represented here qualitatively by a 2-tuple label) is defined by:

$\mathbf{m}_{1} \boxplus \mathbf{m}_{2}=\mathbf{m}_{2} \boxplus \mathbf{m}_{1} \triangleq\left\{x \mid x=s_{1}+s_{2}, s_{1} \in \mathbf{m}_{1}, s_{2} \in \mathbf{m}_{2}\right\}$

where the symbol + means the addition operator on labels and with

$$
\left\{\begin{array}{l}
\inf \left(\mathbf{m}_{1}+\mathbf{m}_{2}\right)=\inf \left(\mathbf{m}_{1}\right)+\inf \left(\mathbf{m}_{2}\right), \\
\sup \left(\mathbf{m}_{1}+\mathbf{m}_{2}\right)=\sup \left(\mathbf{m}_{1}\right)+\sup \left(\mathbf{m}_{2}\right) .
\end{array}\right.
$$

Special case: if a sources of evidence supplies precise information, i.e. $\mathbf{m}$ is a precise 2-tuple, say $\left(L_{k}, \alpha_{k}^{p}\right)$, then

$$
\begin{aligned}
& \left(L_{k}, \alpha_{k}^{p}\right) \boxplus \mathbf{m}_{2}=\mathbf{m}_{2} \boxplus\left(L_{k}, \alpha_{k}^{p}\right) \\
& \quad=\left\{x \mid x=\left(L_{k}, \alpha_{k}^{p}\right)+s_{2}, s_{2} \in \mathbf{m}_{2}\right\}
\end{aligned}
$$

with

$$
\left\{\begin{array}{l}
\inf \left(\left(L_{k}, \alpha_{k}^{p}\right)+\mathbf{m}_{2}\right)=\left(L_{k}, \sigma_{k}^{p}\right)+\inf \left(\mathbf{m}_{2}\right) \\
\sup \left(\left(L_{k}, \alpha_{k}^{p}\right)+\mathbf{m}_{2}\right)=\left(L_{k}, \alpha_{k}^{p}\right)+\sup \left(\mathbf{m}_{2}\right)
\end{array}\right.
$$

Example If 9 labels are used, i.e. $n=9$,

$$
\begin{aligned}
& {\left[\left(L_{1}, 0.1\right),\left(L_{3}, 0.2\right)\right] \boxplus\left[\left(L_{2}, 0.2\right),\left(L_{5}, 0.3\right)\right]} \\
& \quad=\left[\left(L_{3}, 0.3\right),\left(L_{8}, 0.5\right)\right], \\
& L_{3} \boxplus\left[\left(L_{2}, 0.2\right),\left(L_{5}, 0.3\right)\right]=\left[\left(L_{5}, 0.2\right),\left(L_{8}, 0.3\right)\right] .
\end{aligned}
$$

\subsubsection{Subtraction of imprecise 2-tuple labels}

The subtraction operator is defined as follows:

$\mathbf{m}_{1} \boxminus \mathbf{m}_{2} \triangleq\left\{x \mid x=s_{1}-s_{2}, s_{1} \in \mathbf{m}_{1}, s_{2} \in \mathbf{m}_{2}\right\}$

where the symbol - represents the subtraction operator on labels and with

$\left\{\begin{array}{l}\inf \left(\mathbf{m}_{1}-\mathbf{m}_{2}\right)=\inf \left(\mathbf{m}_{1}\right)-\sup \left(\mathbf{m}_{2}\right), \\ \sup \left(\mathbf{m}_{1}-\mathbf{m}_{2}\right)=\sup \left(\mathbf{m}_{1}\right)-\inf \left(\mathbf{m}_{2}\right) .\end{array}\right.$

When $\sup \left(\mathbf{m}_{1}-\mathbf{m}_{2}\right) \leq\left(L_{0}, 0\right)$, one takes $\mathbf{m}_{1} \boxminus \mathbf{m}_{2}=$ $\left(L_{0}, 0\right)$; If $\inf \left(\mathbf{m}_{1}-\mathbf{m}_{2}\right) \leq\left(L_{0}, 0\right), \sup \left(\mathbf{m}_{1}-\mathbf{m}_{2}\right) \geq\left(L_{0}, 0\right)$, then $\mathbf{m}_{1} \boxminus \mathbf{m}_{2}=\left[\left(L_{0}, 0\right), \sup \left(\mathbf{m}_{1}-\mathbf{m}_{2}\right)\right]$; Otherwise, $\mathbf{m}_{1} \boxminus$ $\mathbf{m}_{2}=\left[\inf \left(\mathbf{m}_{1}-\mathbf{m}_{2}\right), \sup \left(\mathbf{m}_{1}-\mathbf{m}_{2}\right)\right]$.

Special case: if one of sources of evidence supplies precise information, i.e. $\mathbf{m}$ is a precise 2-tuple, say $\left(L_{k}, \alpha_{k}^{p}\right)$, then

$$
\left(L_{k}, \alpha_{k}^{p}\right) \boxminus \mathbf{m}_{2}=\left\{x \mid x=\left(L_{k}, \alpha_{k}^{p}\right)-s_{2}, s_{2} \in \mathbf{m}_{2}\right\}
$$


with

$\left\{\begin{array}{l}\inf \left(\left(L_{k}, \alpha_{k}^{p}\right)-\mathbf{m}_{2}\right)=\left(L_{k}, \alpha_{k}^{p}\right)-\sup \left(\mathbf{m}_{2}\right), \\ \sup \left(\left(L_{k}, \alpha_{k}^{p}\right)-\mathbf{m}_{2}\right)=\left(L_{k}, \alpha_{k}^{p}\right)-\inf \left(\mathbf{m}_{2}\right) .\end{array}\right.$

Similarly,

$\mathbf{m}_{1} \boxminus\left(L_{k}, \alpha_{k}^{p}\right)=\left\{x \mid x=s_{1}-\left(L_{k}, \alpha_{k}^{p}\right), s_{1} \in \mathbf{m}_{1}\right\}$

with

$\left\{\begin{array}{l}\inf \left(\mathbf{m}_{1}-\left(L_{k}, \alpha_{k}^{p}\right)\right)=\inf \left(\mathbf{m}_{1}\right)-\left(L_{k}, \alpha_{k}^{p}\right), \\ \sup \left(\mathbf{m}_{1}-\left(L_{k}, \alpha_{k}^{p}\right)\right)=\sup \left(\mathbf{m}_{1}\right)-\left(L_{k}, \alpha_{k}^{p}\right) .\end{array}\right.$

Example If 9 labels are used, i.e. $n=9$,

$\left[\left(L_{2}, 0.2\right),\left(L_{5}, 0.3\right)\right] \boxminus\left[\left(L_{1}, 0.1\right),\left(L_{3}, 0.2\right)\right]$

$=\left[\left(L_{0}, 0\right),\left(L_{4}, 0.2\right)\right]$,

$\left[\left(L_{1}, 0.1\right),\left(L_{3}, 0.2\right)\right] \boxminus\left(L_{5}, 0.3\right)=\left(L_{0}, 0\right)$,

$L_{3} \boxminus\left[\left(L_{2}, 0.2\right),\left(L_{5}, 0.3\right)\right]=\left[\left(L_{0}, 0\right),\left(L_{2}, 0.8\right)\right]$.

\subsubsection{Multiplication of imprecise 2-tuple labels}

The multiplication operator plays also an important role in most of the rules of combinations. The multiplication of imprecise 2-tuple labels is defined as follows:

$\mathbf{m}_{1} \ominus \mathbf{m}_{2}=\mathbf{m}_{2} \bullet \mathbf{m}_{1} \triangleq\left\{x \mid x=s_{1} \times s_{2}, s_{1} \in \mathbf{m}_{1}, s_{2} \in \mathbf{m}_{2}\right\}$

where the symbol $\times$ represents the multiplication operator on labels and with

$\left\{\begin{array}{l}\inf \left(\mathbf{m}_{1} \times \mathbf{m}_{2}\right)=\inf \left(\mathbf{m}_{1}\right) \times \inf \left(\mathbf{m}_{2}\right), \\ \sup \left(\mathbf{m}_{1} \times \mathbf{m}_{2}\right)=\sup \left(\mathbf{m}_{1}\right) \times \sup \left(\mathbf{m}_{2}\right) .\end{array}\right.$

Special case: if one of sources of evidence supplies precise information, i.e. $\mathbf{m}$ is a precise 2 -tuple, say $\left(L_{k}, \alpha_{k}^{p}\right)$, then

$$
\begin{aligned}
& \left(L_{k}, \alpha_{k}^{p}\right) \triangleright \mathbf{m}_{2}=\mathbf{m}_{2} \bullet\left(L_{k}, \alpha_{k}^{p}\right) \\
& \quad=\left\{x \mid x=\left(L_{k}, \alpha_{k}^{p}\right) \times s_{2}, s_{2} \in \mathbf{m}_{2}\right\}
\end{aligned}
$$

with

$$
\left\{\begin{array}{l}
\inf \left(\left(L_{k}, \alpha_{k}^{p}\right) \times \mathbf{m}_{2}\right)=\left(L_{k}, \alpha_{k}^{p}\right) \times \inf \left(\mathbf{m}_{2}\right) \\
\sup \left(\left(L_{k}, \alpha_{k}^{p}\right) \times \mathbf{m}_{2}\right)=\left(L_{k}, \alpha_{k}^{p}\right) \times \sup \left(\mathbf{m}_{2}\right) .
\end{array}\right.
$$

Example If 9 labels are used, i.e. $n=9$,

$$
\begin{aligned}
& \quad\left[\left(L_{1}, 0.1\right),\left(L_{3}, 0.2\right)\right] \odot\left[\left(L_{2}, 0.2\right),\left(L_{5}, 0.3\right)\right] \\
& \quad=\left[\left(L_{1}, 0.838\right),\left(L_{2}, 0.684\right)\right], \\
& L_{3} \odot\left[\left(L_{2}, 0.2\right),\left(L_{5}, 0.3\right)\right]=\left[\left(L_{1}, 0.46\right),\left(L_{2}, 0.59\right)\right] .
\end{aligned}
$$

3.3.4 Division of imprecise 2-tuple labels

The division operator is also necessary in some combinations rules (like in Dempster's rule or PCR5 by example). So we propose the following division operator for imprecise 2-tuple labels based on division of sets introduced in [2]:

If $\mathbf{m}_{2} \neq\left(L_{0}, 0\right)$, then

$\mathbf{m}_{1} \square \mathbf{m}_{2} \triangleq\left\{x \mid x=s_{1} \div s_{2}, s_{1} \in \mathbf{m}_{1}, s_{2} \in \mathbf{m}_{2}\right\}$

where the symbol $\div$ represents the division operator on labels and with

$\left\{\begin{array}{l}\inf \left(\mathbf{m}_{1} \div \mathbf{m}_{2}\right)=\inf \left(\mathbf{m}_{1}\right) \div \sup \left(\mathbf{m}_{2}\right) \\ \sup \left(\mathbf{m}_{1} \div \mathbf{m}_{2}\right)=\sup \left(\mathbf{m}_{1}\right) \div \inf \left(\mathbf{m}_{2}\right)\end{array}\right.$

when $\sup \left(\mathbf{m}_{1}\right) \div \inf \left(\mathbf{m}_{2}\right) \leq L_{n+1}$. Otherwise we take $\sup \left(\mathbf{m}_{1} \div \mathbf{m}_{2}\right)=L_{n+1}$.

Special case: if one of sources of evidence supplies precise information, i.e. $\mathbf{m}$ is a precise 2-tuple, say $\left(L_{k}, \alpha_{k}^{p}\right) \neq$ $\left(L_{0}, 0\right)$, then

$\left(L_{k}, \alpha_{k}^{p}\right) \square \mathbf{m}_{2}=\left\{x \mid x=\left(L_{k}, \alpha_{k}^{p}\right) \div s_{2}, s_{2} \in \mathbf{m}_{2}\right\}$

with

$\left\{\begin{array}{l}\inf \left(\left(L_{k}, \alpha_{k}^{p}\right) \div \mathbf{m}_{2}\right)=\left(L_{k}, \alpha_{k}^{p}\right) \div \sup \left(\mathbf{m}_{2}\right) \\ \sup \left(\left(L_{k}, \alpha_{k}^{p}\right) \div \mathbf{m}_{2}\right)=\left(\left(L_{k}, \alpha_{k}^{p}\right) \div \inf \left(\mathbf{m}_{2}\right)\right.\end{array}\right.$

Similarly,

$\mathbf{m}_{1} \square\left(L_{k}, \alpha_{k}^{p}\right)=\left\{x \mid x=s_{1} \div\left(L_{k}, \alpha_{k}^{p}\right), s_{1} \in \mathbf{m}_{1}\right\}$

with

$\left\{\begin{array}{l}\inf \left(\mathbf{m}_{1} \div\left(L_{k}, \alpha_{k}^{p}\right)\right)=\inf \left(\mathbf{m}_{2}\right) \div\left(L_{k}, \alpha_{k}^{p}\right) \\ \sup \left(\mathbf{m}_{1} \div\left(L_{k}, \alpha_{k}^{p}\right)\right)=\sup \left(\mathbf{m}_{2}\right) \div\left(L_{k}, \alpha_{k}^{p}\right)\end{array}\right.$

Example If 9 labels are used, i.e. $n=9$,

$$
\begin{aligned}
& \quad\left[\left(L_{1}, 0.1\right),\left(L_{3}, 0.2\right)\right] \square\left[\left(L_{2}, 0.2\right),\left(L_{5}, 0.3\right)\right] \\
& \quad=\left[\left(L_{2}, 0.085\right),\left(L_{10}, 0\right)\right], \\
& L_{3} \square\left[\left(L_{2}, 0.2\right),\left(L_{5}, 0.3\right)\right]=\left[\left(L_{7},-0.617\right),\left(L_{10}, 0\right)\right], \\
& {\left[\left(L_{2}, 0.2\right),\left(L_{5}, 0.3\right)\right] \square L_{3}=\left[\left(L_{6}, 0\right),\left(L_{10}, 0\right)\right] .}
\end{aligned}
$$

\section{Fusion of qualitative beliefs}

4.1 Fusion of precise qualitative beliefs

From the 2-tuple linguistic representation model of qualitative beliefs and the previous operators on 2-tuple labels, we are now able to extend the $D S m C, P C R 5$ and even Dempster's (DS) fusion rules into the qualitative domain following the track of our previous works [3, 14, 23]. We denote 
$q_{2} m(\cdot)$ the qualitative belief mass/assignment (qba) based on 2-tuple representation in order to make a difference with the qba $q_{1} m(\cdot)$ based on 1-tuple (classical/pure) linguistic labels and $q_{e} m(\cdot)$ based on qualitative enriched linguistic labels[14]. Mathematically, $q_{2} m(\cdot)$ expressed by a given source/body of evidence $S$ is defined as a mapping function $q_{2} m(\cdot): G^{\Theta} \rightarrow L \times \alpha$ such that:

$q_{2} m(\emptyset)=\left(L_{0}, 0\right) \quad$ and $\quad \sum_{A \in G^{\Theta}} q_{2} m(A)=\left(L_{n+1}, 0\right)$.

From the expressions of quantitative $D S m C$ (2), PCR5 (3) and Dempster's $(D S)$ [20] fusion rules and from the operators on 2-tuple labels, we can define the classical qualitative combination or proportional redistribution rules ( $q_{2} D S m C$ and $q_{2} P C R 5$ ) for dealing with 2-tuple linguistic labels $\left(L_{i}, \sigma_{i}^{p}\right)$. This is done as follows:

- when working with the free $D S m$ model of the frame $\Theta$ : $q_{2} m_{D S m C}(\emptyset)=\left(L_{0}, 0\right)$ and $\forall X \in D^{\Theta} \backslash\{\varnothing\}$

$$
q_{2} m_{D S m C}(X)=\sum_{\substack{X_{1}, X_{2} \in D^{\Theta} \\ X_{1} \cap X_{2}=X}} q_{2} m_{1}\left(X_{1}\right) q_{2} m_{2}\left(X_{2}\right)
$$

- when working with Shafer's or hybrid model of the frame $\Theta: q_{2} m_{P C R 5}(\emptyset)=\left(L_{0}, 0\right)$ and $\forall X \in G^{\Theta} \backslash\{\emptyset\}$

$$
\begin{aligned}
& q_{2} m_{P C R 5}(X) \\
& =q_{2} m_{12}(X)+\sum_{\substack{Y \in G^{\Theta} \backslash\{X\} \\
X \cap Y=\emptyset}}\left[\frac{q_{2} m_{1}(X)^{2} q_{2} m_{2}(Y)}{q_{2} m_{1}(X)+q_{2} m_{2}(Y)}\right. \\
& \left.\quad+\frac{q_{2} m_{2}(X)^{2} q_{2} m_{1}(Y)}{q_{2} m_{2}(X)+q_{2} m_{1}(Y)}\right]
\end{aligned}
$$

where $q_{2} m_{12}(X)$ corresponds to the qualitative conjunctive consensus.

It is important to note that addition, product and division operators involved in formulas (32) and (33) are 2-tuple operators defined in the previous section. These rules can be easily extended for the qualitative fusion of $k>2$ sources of evidence. The formulas (32) and (33) are well justified since every 2-tuple $\left(L_{i}, \sigma_{i}^{p}\right)$ can be mapped into a unique numerical value corresponding to it which makes the qualitative fusion rules $q_{2} D S m C$ and $q_{2} P C R 5$ equivalent to the corresponding numerical fusion rules $D S m C$ and $P C R 5$.

Theorem 1 (Normalization) If $\sum_{A \in G^{\Theta}} q_{2} m(A)=$ $\left(L_{n+1}, 0\right)$, then $\sum_{A \in G^{\Theta}} q_{2} m_{D S m C}(A)=\left(L_{n+1}, 0\right)$, and $\sum_{A \in G^{\Theta}} q_{2} m_{P C R 5}(A)=\left(L_{n+1}, 0\right)$.

Proof Let's assume that there is a frame of discernment $\Theta$ which includes several focal elements. According to $D S m$ model, one defines its hyper-power set $D^{\Theta}, A_{i} \in D^{\Theta}, i=$
$\{1,2, \ldots, n\}$. There exist $k$ evidential sources with qualitative belief mass $a_{i j}, i \in\{1,2, \ldots, k\}, j \in\{1,2, \ldots, n\}$. According to the premise, i.e. $\sum_{A \in G^{\Theta}} q_{2} m(A)=\left(L_{n+1}, 0\right)$, that is, $\sum_{j \in\{1,2, \ldots, n\}} a_{i j}=\left(L_{n+1}, 0\right)$. According to (16) and the characteristics of Product operator,

$\prod_{i \in\{1,2, \ldots, k\}} \sum_{j \in\{1,2, \ldots, n\}} a_{i j}=\prod_{i \in\{1,2, \ldots, k\}}\left(L_{n+1}, 0\right)=\left(L_{n+1}, 0\right)$

because

$$
\begin{aligned}
& q_{2} m_{D S m C}(X) \\
& \quad=\sum_{\substack{X_{1}, X_{2}, \ldots, X_{k} \in D^{\Theta} \\
X_{1} \cap X_{2} \cdots X_{k}=X}} q_{2} m_{1}\left(X_{1}\right) q_{2} m_{2}\left(X_{2}\right) \cdots q_{2} m_{k}\left(X_{k}\right) \\
& =\prod_{i \in\{1,2, \ldots, k\}} \sum_{j \in\{1,2, \ldots, n\}} a_{i j}=\left(L_{n+1}, 0\right) .
\end{aligned}
$$

Moreover, since $q P C R 5$ redistributes proportionally the partial conflicting mass to the elements involved in the partial conflict by considering the canonical form of the partial conflict, the total sum of all qualitative belief mass after redistribution doesn't change and therefore it is equal to $\left(L_{n+1}, 0\right)$. This completes the proof.

Similarly, Dempster's rule (DS) can be extended for dealing with 2-tuple linguistic labels by taking $q_{2} m_{D S}(\emptyset)=$ $\left(L_{0}, 0\right)$ and $\forall A \in 2^{\Theta} \backslash\{\emptyset\}$

$$
q_{2} m_{D S}(A)=\frac{\sum_{\substack{X, Y \in 2^{\Theta} \\ X \cap Y=A}} q_{2} m_{1}(X) q_{2} m_{2}(Y)}{\left(L_{n+1}, 0\right)-\sum_{\substack{X, Y \in 2^{\Theta} \\ X \cap Y=\emptyset}} q_{2} m_{1}(X) q_{2} m_{2}(Y)} .
$$

\subsection{Fusion of imprecise qualitative beliefs}

Let's consider $k$ sources of evidences providing imprecise qualitative belief assignments/masses $\mathbf{m}_{i j}$ defined on $G^{\Theta}$ with $\left|G^{\Theta}\right|=d$. We denote by $m_{i j}$ central value of the label provided by the source no. $i(1 \leq i \leq k)$ for the element $X_{j} \in G^{\Theta}, 1 \leq j \leq d$. For example with qualitative interval-valued beliefs, $\mathbf{m}_{i j}=\left[m_{i j}-\epsilon_{i j}, m_{i j}+\epsilon_{i j}\right] \epsilon$ $\left[\left(L_{0}, 0\right),\left(L_{n+1}, 0\right)\right]$, where $\left(L_{0}, 0\right) \leq \epsilon_{i j} \leq L_{n+1}$. More generally, $\mathbf{m}_{i j}$ can be either an union of open intervals, or of closed intervals, or of semi-open intervals.

The set of imprecise qualitative belief masses provided by the sources of evidences can be represented/characterized by the following belief mass matrices with

$$
\inf (\mathbf{M})=\left[\begin{array}{llll}
m_{11}-\epsilon_{11} & m_{12}-\epsilon_{12} & \cdots & m_{1 d}-\epsilon_{1 d} \\
m_{21}-\epsilon_{21} & m_{22}-\epsilon_{22} & \cdots & m_{2 d}-\epsilon_{2 d} \\
\cdots & \cdots & \cdots & \cdots \\
m_{k 1}-\epsilon_{k 1} & m_{k 2}-\epsilon_{k 2} & \cdots & m_{k d}-\epsilon_{k d}
\end{array}\right],
$$


$\sup (\mathbf{M})=\left[\begin{array}{llll}m_{11}+\epsilon_{11} & m_{12}+\epsilon_{12} & \cdots & m_{1 d}+\epsilon_{1 d} \\ m_{21}+\epsilon_{21} & m_{22}+\epsilon_{22} & \cdots & m_{2 d}+\epsilon_{2 d} \\ \cdots & \cdots & \cdots & \cdots \\ m_{k 1}+\epsilon_{k 1} & m_{k 2}+\epsilon_{k 2} & \cdots & m_{k d}+\epsilon_{k d}\end{array}\right]$.

All the previous qualitative fusion rules working with precise 2-tuple labels can be extended directly for dealing with imprecise 2-tuple labels by replacing precise operators on 2-tuple labels by their counterparts for imprecise 2-tuple labels. We just here present the extensions of $D S m C, P C R 5$ and $D S$ rules of combinations. The extensions of other combination rules (DSmH, Dubois \& Prade's, Yager's, etc) can be done easily in a similar way and will not be reported here.

\section{- The DSmC fusion of imprecise qualitative beliefs}

The $D S m$ classical combinational rule of $k \geq 2$ imprecise qualitative beliefs is defined for the free DSm model of the frame $\Theta$, i.e. $G^{\Theta}=D^{\Theta}$ as follows: $q_{2} m_{D S m C}^{I}(\emptyset)=$ $\left(L_{0}, 0\right)$ and $\forall X \in D^{\Theta} \backslash\{\varnothing\}$

$$
q_{2} \mathbf{m}_{D S m C}^{I}(X)=\sum_{\substack{X_{1}, X_{2}, \ldots, X_{k} \in D^{\Theta} \\ X_{1} \cap X_{2}, \ldots, \cap X_{k}=X}} \prod_{i=1}^{k} q_{2} \mathbf{m}_{i}\left(X_{i}\right) .
$$

- The PCR5 fusion of imprecise qualitative beliefs

When working with Shafer's or DSm hybrid models of the frame $\Theta$, the PCR5 combinational rule of two imprecise qualitative beliefs is defined by: $q_{2} m_{P C R 5}^{I}(\emptyset)=$ $\left(L_{0}, 0\right)$ and $\forall X \in G^{\Theta} \backslash\{\emptyset\}$

$$
\begin{aligned}
q_{2} \mathbf{m}_{P C R 5}^{I}(X) & \\
= & q_{2} \mathbf{m}_{12}^{I}(X)+\sum_{\substack{Y \in G^{\Theta} \backslash\{X\} \\
X \cap Y=\emptyset}}\left[\frac{q_{2} \mathbf{m}_{1}(X)^{2} q_{2} \mathbf{m}_{2}(Y)}{q_{2} \mathbf{m}_{1}(X)+q_{2} \mathbf{m}_{2}(Y)}\right. \\
& \left.+\frac{q_{2} \mathbf{m}_{2}(X)^{2} q_{2} \mathbf{m}_{1}(Y)}{q_{2} \mathbf{m}_{2}(X)+q_{2} \mathbf{m}_{1}(Y)}\right]
\end{aligned}
$$

where $q_{2} \mathbf{m}_{12}^{I}(X)$ corresponds to the imprecise qualitative conjunctive consensus defined by

$$
q_{2} \mathbf{m}_{12}^{I}(X)=\sum_{\substack{X_{1}, X_{2} \in G^{\Theta} \\ X_{1} \cap X_{2}=X}} q_{2} \mathbf{m}_{1}\left(X_{1}\right) q_{2} \mathbf{m}_{2}\left(X_{2}\right) .
$$

- Dempster's fusion of imprecise qualitative beliefs

Dempster's rule can also be directly extended for dealing with imprecise qualitative beliefs by taking $q_{2} \mathbf{m}_{D S}(\emptyset)=\left(L_{0}, 0\right)$ and $\forall A \in 2^{\Theta} \backslash\{\emptyset\}$

$$
q_{2} \mathbf{m}_{D S}^{I}(A)=\frac{\sum_{\substack{X, Y \in 2 \\ X \cap Y=A}} q_{2} \mathbf{m}_{1}(X) q_{2} \mathbf{m}_{2}(Y)}{\left(L_{n+1}, 0\right)-\sum_{\substack{X, Y \in 2^{\Theta} \\ X \cap Y=\emptyset}} q_{2} \mathbf{m}_{1}(X) q_{2} \mathbf{m}_{2}(Y)} .
$$

Theorem 2 The following equality holds

$q_{2} \boldsymbol{m}_{D S m C}^{I}(X)=\left[\inf \left(q_{2} \boldsymbol{m}_{D S m C}^{I}(X)\right), \sup \left(q_{2} \boldsymbol{m}_{D S m C}^{I}(X)\right)\right]$

with

$$
\inf \left(q_{2} \boldsymbol{m}_{D S m C}^{I}(X)\right)=\sum_{\substack{X_{1}, X_{2}, \ldots, X_{k} \in D^{\Theta} \\ X_{1} \cap X_{2}, \ldots, \cap X_{k}=X}} \prod_{i=1}^{k} \inf \left(q_{2} \boldsymbol{m}_{i}\left(X_{i}\right)\right),
$$

$$
\sup \left(q_{2} \boldsymbol{m}_{D S m C}^{I}(X)\right)=\sum_{\substack{X_{1}, X_{2}, \ldots, X_{k} \in D^{\Theta} \\ X_{1} \cap X_{2}, \ldots, X_{k}=X}} \prod_{i=1}^{k} \sup \left(q_{2} \boldsymbol{m}_{i}\left(X_{i}\right)\right) .
$$

Proof Let's assume $\inf \left(q_{2} \mathbf{m}_{i}\left(X_{j}\right)\right)$ and $\sup \left(q_{2} \mathbf{m}_{i}\left(X_{j}\right)\right)$ $(1 \leq i \leq k)$ be represented by $a_{i j} \in \inf (\mathbf{M})$ and $b_{i j} \in \sup (\mathbf{M})$ with $a_{i j} \leq b_{i j}$ ( $\leq$ represents here a qualitative order). For any label $c_{m j} \in\left[a_{m j}, b_{m j}\right]$, one has

$$
\sum_{\substack{X_{1}, X_{2}, \ldots, X_{k} \in D^{\Theta} \\ X_{1} \cap X_{2}, \ldots, \cap X_{k}=X}} \prod_{i=1}^{k} a_{i j} \leq \sum_{\substack{X_{1}, X_{2}, \ldots, X_{k} \in D^{\Theta} \\ X_{1} \cap X_{2}, \ldots, \cap X_{k}=X}} \prod_{i=1, i \neq m}^{k} a_{i j} c_{m j}
$$

and also

$$
\sum_{\substack{X_{1}, X_{2}, \ldots, X_{k} \in D^{\Theta} \\ X_{1} \cap X_{2}, \ldots, \cap X_{k}=X}} \prod_{i=1, i \neq m}^{k} a_{i j} c_{m j} \leq \sum_{\substack{X_{1}, X_{2}, \ldots, X_{k} \in D^{\Theta} \\ X_{1} \cap X_{2}, \ldots, \cap X_{k}=X}} \prod_{i=1}^{k} b_{i j} .
$$

Therefore, $\quad q_{2} \mathbf{m}_{D S m C}^{I}(X)=\left[\inf \left(q_{2} \mathbf{m}_{D S m C}^{I}(X)\right)\right.$, $\left.\sup \left(q_{2} \mathbf{m}_{D S m C}^{I}(X)\right)\right]$ which completes the proof.

Therefore, this theorem supplies with a terse way to combine imprecise qualitative belief in DSmT framework. That is, we can respectively compute the upper and lower border of imprecise qualitative beliefs with $q_{2} D S m C$ given in (32).

When working with Shafer's or hybrid model of the frame $\Theta: q_{2} m_{P C R 5}^{I}(\emptyset)=\left(L_{0}, 0\right)$ and $\forall X \in G^{\Theta} \backslash\{\emptyset\}$. The $P C R 5$ of imprecise qualitative beliefs $\left(q_{2}^{I} P C R 5\right)$ is given as follows:

$$
\begin{aligned}
& \sup \left(q_{2} \mathbf{m}_{P C R 5}^{I}(X)\right) \\
& =\sup \left(q_{2} \mathbf{m}_{12}(X)\right) \\
& \quad+\sum_{Y \in G^{\Theta} \backslash\{X\} X \cap Y=\emptyset}\left[\frac{\sup \left(q_{2} \mathbf{m}_{1}(X)^{2} q_{2} \mathbf{m}_{2}(Y)\right)}{\inf \left(q_{2} \mathbf{m}_{1}(X)+q_{2} \mathbf{m}_{2}(Y)\right)}\right. \\
& \left.\quad+\frac{\sup \left(q_{2} \mathbf{m}_{2}(X)^{2} q_{2} \mathbf{m}_{1}(Y)\right)}{\inf \left(q_{2} \mathbf{m}_{2}(X)+q_{2} \mathbf{m}_{1}(Y)\right)}\right] \\
& \inf \left(q_{2} \mathbf{m}_{P C R 5}^{I}(X)\right) \\
& =\inf \left(q_{2} \mathbf{m}_{12}(X)\right)
\end{aligned}
$$




$$
\begin{aligned}
& +\sum_{Y \in G^{\Theta} \backslash\{X\} X \cap Y=\emptyset}\left[\frac{\inf \left(q_{2} \mathbf{m}_{1}(X)^{2} q_{2} \mathbf{m}_{2}(Y)\right)}{\sup \left(q_{2} \mathbf{m}_{1}(X)+q_{2} \mathbf{m}_{2}(Y)\right)}\right. \\
& \left.+\frac{\inf \left(q_{2} \mathbf{m}_{2}(X)^{2} q_{2} \mathbf{m}_{1}(Y)\right)}{\sup \left(q_{2} \mathbf{m}_{2}(X)+q_{2} \mathbf{m}_{1}(Y)\right)}\right]
\end{aligned}
$$

where $q_{2} \mathbf{m}_{12}(X)$ corresponds to $q_{2}^{I} D S m T$ of the conjunctive consensus.

\section{Examples of fusion of qualitative beliefs}

\subsection{Example of fusion of precise qualitative beliefs}

Let's consider an investment corporation which has to choose one project among three proposals $\Theta=\left\{\theta_{1}, \theta_{2}, \theta_{3}\right\}$ based on two consulting/expert reports. The linguistic labels used by the experts are among the following ones: $I \mapsto \mathrm{Im}$ possible, EU $\mapsto$ Extremely-Unlikely, VLC $\mapsto$ Very-LowChance, LLC $\mapsto$ Little-Low-Chance, $\mathrm{SC} \mapsto$ Small-Chance, $\mathrm{IM} \mapsto$ IT-May, MC $\mapsto$ Meanful-Chance, LBC $\mapsto$ LittleBig-Chance, $\mathrm{BC} \mapsto$ Big-Chance, ML $\mapsto$ Most-likely, $\mathrm{C} \mapsto$ Certain. So, we consider the following ordered set $L$ (with $|L|=n=9$ ) of linguistic labels

$$
\begin{aligned}
L \triangleq & \left\{L_{0} \equiv I, L_{1} \equiv E U, L_{2} \equiv V L C, L_{3} \equiv L L C, L_{4} \equiv S C,\right. \\
& L_{5} \equiv I M, L_{6} \equiv M C, L_{7} \equiv L B C, L_{8} \equiv B C, \\
& \left.L_{9} \equiv M L, L_{10} \equiv C\right\} .
\end{aligned}
$$

The qualitative belief assignments/masses provided by the sources/experts are assumed to be given according to Table 1.

When working with the free DSm model and applying the qualitative $D S m C$ combinational rule (32), we obtain:

$$
\begin{array}{ll}
q_{2} m_{D S m C}\left(\theta_{1}\right) & =\left(L_{3}, 0.85\right), \\
q_{2} m_{D S m C}\left(\theta_{2}\right) & =\left(L_{1}, 0.433\right), \\
q_{2} m_{D S m C}\left(\theta_{3}\right) & =\left(L_{1}, 0.13\right), \\
q_{2} m_{D S m C}\left(\theta_{1} \cap \theta_{2}\right) & =\left(L_{3}, 0.747\right), \\
q_{2} m_{D S m C}\left(\theta_{1} \cap \theta_{3}\right) & =\left(L_{3}, 0.253\right), \\
q_{2} m_{D S m C}\left(\theta_{2} \cap \theta_{3}\right) & =\left(L_{2}, 0.587\right) .
\end{array}
$$

Table 1 Precise qualitative belief assignments given by the sources

\begin{tabular}{lll}
\hline & Source 1 & Source 2 \\
\hline$\theta_{1}$ & $m_{1}\left(\theta_{1}\right)=\left(L_{5}, 0.7\right)$ & $m_{2}\left(\theta_{1}\right)=\left(L_{5}, 0\right)$ \\
$\theta_{2}$ & $m_{1}\left(\theta_{2}\right)=\left(L_{3}, 0.3\right)$ & $m_{2}\left(\theta_{2}\right)=\left(L_{3}, 0.9\right)$ \\
$\theta_{3}$ & $m_{1}\left(\theta_{3}\right)=\left(L_{3}, 0\right)$ & $m_{2}\left(\theta_{3}\right)=\left(L_{3}, 0.1\right)$ \\
\hline
\end{tabular}

We can verify the validity of the Theorem 1, i.e. $\sum_{A \in D^{\Theta}} q_{2} m(A)=\left(L_{10}, 0\right)$, which proves that is $q_{2} m_{D S m C}($.$) is normalized.$

Now, let's assume that Shafer's model holds for $\Theta$. In this case the sets $\theta_{1} \cap \theta_{2}, \theta_{1} \cap \theta_{3}, \theta_{2} \cap \theta_{3}$ must be empty and the qualitative conflicting masses $q_{2} m_{D S m C}\left(\theta_{1} \cap \theta_{2}\right)$, $q_{2} m_{D S m C}\left(\theta_{1} \cap \theta_{3}\right)$ and $q_{2} m_{D S m C}\left(\theta_{2} \cap \theta_{3}\right)$ need to be redistributed to the sets involved in these conflicts according to (33) if the PCR5 fusion rule is used. So, with PCR5 one gets:

$$
\begin{aligned}
q_{2} m_{P C R 5}\left(\theta_{1}\right)= & q_{2} m_{D S m C}\left(\theta_{1}\right)+q_{2} m_{x A 1}\left(\theta_{1}\right) \\
& +q_{2} m_{x B 1}\left(\theta_{1}\right)+q_{2} m_{x A 2}\left(\theta_{1}\right) \\
& +q_{2} m_{x B 2}\left(\theta_{1}\right) \\
= & \left(L_{6}, 0.684\right), \\
q_{2} m_{P C R 5}\left(\theta_{2}\right)= & q_{2} m_{D S m C}\left(\theta_{2}\right)+q_{2} m_{y A 1}\left(\theta_{2}\right) \\
& +q_{2} m_{y B 1}\left(\theta_{2}\right)+q_{2} m_{x A 3}\left(\theta_{2}\right) \\
& +q_{2} m_{x B 3}\left(\theta_{2}\right) \\
= & \left(L_{2}, 0.0264\right), \\
q_{2} m_{P C R 5}\left(\theta_{3}\right)= & q_{2} m_{D S m C}\left(\theta_{3}\right)+q_{2} m_{y A 2}\left(\theta_{3}\right) \\
& +q_{2} m_{y B 2}\left(\theta_{3}\right)+q_{2} m_{y A 3}\left(\theta_{3}\right) \\
& +q_{2} m_{y B 3}\left(\theta_{3}\right) \\
= & \left(L_{3}, 0.289\right) .
\end{aligned}
$$

Because $q_{2} m_{P C R 5}\left(\theta_{1}\right)$ is larger than $q_{2} m_{P C R 5}\left(\theta_{2}\right)$ and $q_{2} m_{P C R 5}\left(\theta_{3}\right)$, the investment corporation will choose the first project to invest.

Now, if we prefer to use the extension of Dempter's rule of combination given by the formula (38), the total qualitative conflicting mass is $q K_{\text {total }}=q_{2} m_{D S m C}\left(\theta_{1} \cap \theta_{2}\right)+$ $q_{2} m_{D S m C}\left(\theta_{1} \cap \theta_{3}\right)+q_{2} m_{D S m C}\left(\theta_{3} \cap \theta_{2}\right)=\left(L_{7}, 0.587\right)$, and so we obtain:

$$
\begin{aligned}
q_{2} m_{D S}(\emptyset) & \triangleq\left(L_{0}, 0\right), \\
q_{2} m_{D S}\left(\theta_{1}\right) & =\frac{q_{2} m_{D S m C}\left(\theta_{1}\right)}{L_{10}-q K_{\text {total }}}=\frac{\left(L_{3}, 0.85\right)}{L_{10}-\left(L_{7}, 0.587\right)} \\
& =\left(L_{6}, 0.006133\right), \\
q_{2} m_{D S}\left(\theta_{2}\right) & =\frac{q_{2} m_{D S m C}\left(\theta_{2}\right)}{L_{10}-q K_{\text {total }}}=\frac{\left(L_{1}, 0.413\right)}{L_{10}-\left(L_{7}, 0.587\right)} \\
& =\left(L_{2}, 0.419292\right),
\end{aligned}
$$

$q_{2} m_{D S}\left(\theta_{3}\right)=\frac{q_{2} m_{D S m C}\left(\theta_{3}\right)}{L_{10}-q K_{\text {total }}}=\frac{\left(L_{1}, 0.13\right)}{L_{10}-\left(L_{7}, 0.587\right)}$

$$
=\left(L_{3}, 0.574575\right) \text {. }
$$

We see that $q_{2} m_{D S}\left(\theta_{1}\right)$ is larger than $q_{2} m_{D S}\left(\theta_{2}\right)$ and $q_{2} m_{D S}\left(\theta_{3}\right)$, so the first project is also chosen to invest. 
The final decision is same to the previous one obtained by $q_{2} P C R 5$. However, when the total conflict becomes nearer and nearer to $L_{10}$, then $q_{2} D S$ formula will become invalid. If we adopt the simple arithmetic mean method, the results of the fusion are:

$$
\begin{array}{ll}
\theta_{1}: & \frac{\left(L_{5}, 0.7\right)+\left(L_{5}, 0\right)}{2}=\left(L_{5}, 0.35\right), \\
\theta_{2}: & \frac{\left(L_{3}, 0.3\right)+\left(L_{3}, 0.9\right)}{2}=\left(L_{3}, 0.6\right), \\
\theta_{3}: & \frac{\left(L_{3}, 0\right)+\left(L_{3}, 0.1\right)}{2}=\left(L_{3}, 0.05\right) .
\end{array}
$$

According to the above results, we easily know which project will be chosen to invest. Though arithmetic mean method is the simplest method among three methods, for some complex problems, it can provide unsatisfactory results since it is not neutral with respect to the introduction of a total ignorant source in the fusion process. This method can also be ill adapted to some particular problems. For example, one also investigates the possibility of investment in two projects together, i.e. $\theta_{i} \cap \theta_{j} \neq \emptyset$. However, the corporation only choose one of them to invest. How to do it in this case with simple arithmetic mean method? It is more easy to take decision from $q_{2} P C R 5$ (.).

If all qualitative masses involved in the fusion are normalized, no matter what qualitative fusion rule we use the normalization is kept (i.e. the result will also be a normalized mass).

\subsection{Example of fusion of imprecise qualitative beliefs}

Let's consider again the previous example with imprecise qualitative beliefs provided by the sources according to Table 2 .

If one works with the free DSm model for the frame $\Theta$, one gets from (35) and the theorem 2 the following results:

$$
\begin{aligned}
& q_{2} m_{D S m C}^{I}\left(\theta_{1}\right)=\left[\left(L_{3}, 0.85\right),\left(L_{3}, 0.138\right)\right], \\
& q_{2} m_{D S m C}^{I}\left(\theta_{2}\right)=\left[\left(L_{1}, 0.433\right),\left(L_{1}, 0.001\right)\right], \\
& q_{2} m_{D S m C}^{I}\left(\theta_{3}\right)=\left[\left(L_{1}, 0.13\right),\left(L_{2}, 0.71\right)\right], \\
& q_{2} m_{D S m C}^{I}\left(\theta_{1} \cap \theta_{2}\right)=\left[\left(L_{3}, 0.747\right),\left(L_{4}, 0.571\right)\right], \\
& q_{2} m_{D S m C}^{I}\left(\theta_{1} \cap \theta_{3}\right)=\left[\left(L_{3}, 0.253\right),\left(L_{4}, 0.088\right)\right], \\
& q_{2} m_{D S m C}^{I}\left(\theta_{2} \cap \theta_{3}\right)=\left[\left(L_{2}, 0.587\right),\left(L_{3}, 0.729\right)\right] .
\end{aligned}
$$

Table 2 Imprecise qualitative belief assignments given by the sources

\begin{tabular}{lll}
\hline & Source 1 & Source 2 \\
\hline$\theta_{1}$ & $m_{1}\left(\theta_{1}\right)=\left[\left(L_{5}, 0.7\right),\left(L_{6}, 0.7\right)\right]$ & $m_{2}\left(\theta_{1}\right)=\left[\left(L_{5}, 0\right),\left(L_{6}, 0.6\right)\right]$ \\
$\theta_{2}$ & $m_{1}\left(\theta_{2}\right)=\left[\left(L_{3}, 0.3\right),\left(L_{4}, 0.3\right)\right]$ & $m_{2}\left(\theta_{2}\right)=\left[\left(L_{2}, 0.01\right),\left(L_{3}, 0.3\right)\right]$ \\
$\theta_{3}$ & $m_{1}\left(\theta_{3}\right)=\left[\left(L_{3}, 0\right),\left(L_{5}, 0.7\right)\right]$ & $m_{2}\left(\theta_{3}\right)=\left[\left(L_{3}, 0.1\right),\left(L_{3}, 0\right)\right]$ \\
\hline
\end{tabular}

If one works with Shafer's model for the frame $\Theta$ (i.e. all elements of $\Theta$ are assumed exclusive), then the imprecise qualitative conflicting masses $q_{2} m_{D S m C}^{I}\left(\theta_{1} \cap \theta_{2}\right)$, $q_{2} m_{D S m C}^{I}\left(\theta_{1} \cap \theta_{3}\right)$ and $q_{2} m_{D S m C}^{I}\left(\theta_{2} \cap \theta_{3}\right)$ need to be redistributed to elements involved in these conflicts if PCR5 is used. In such case and from (36) and the Theorem 2, one gets:

$q_{2} m_{P C R 5}^{I}\left(\theta_{1}\right)=\left[\left(L_{5}, 0.2036\right),\left(L_{9}, 0.8140\right)\right]$,

$q_{2} m_{P C R 5}^{I}\left(\theta_{2}\right)=\left[\left(L_{2}, 0.2909\right),\left(L_{4}, 0.089\right)\right]$,

$q_{2} m_{P C R 5}^{I}\left(\theta_{3}\right)=\left[\left(L_{3}, 0.3308\right),\left(L_{6}, 0.8888\right)\right]$.

From the values of $q_{2} m_{P C R 5}^{I}($.$) , one will choose the$ project $\theta_{1}$ as final decision. It is interesting to note that $q_{2} D S m C$ and $q_{2} P C R 5$ can be interpreted as special case (lower bounds) of $q_{2}^{I} D S m C$ and $q_{2}^{I} P C R 5$.

The approach proposed in this work for combining imprecise qualitative beliefs presents the following properties:

(1) If one utilizes the $q_{2}$-operators on 2-tuples without doing any approximation in the calculations one gets an exact qualitative result, while working on 1-tuples we round the qualitative result so we get approximations. Thus addition and multiplication operators on 2-tuple are truly commutative and associative contrariwise to addition and multiplication operators on 1-tuples. Actually, our new representation deals directly with exact qualitative (refined) values of the labels, which can be explained well by the DSm Field and Linear Algebra of Refined Labels (DSm-FLARL) presented in [24]. In DSm-FLARL we get the exact qualitative result.

(2) Since the 2-tuples $\left\{\left(L_{0}, \sigma_{0}^{p}\right), \ldots,\left(L_{n+1}, \sigma_{n+1}^{p}\right)\right\}$ express actually continuous qualitative beliefs, they are equivalent to real numbers. So all quantitative fusion rules (and even the belief conditioning rules) can work directly using this qualitative framework. The imprecise qualitative DSmC and PCR5 fusion rules can deal easily and efficiently with imprecise belief structures, which are usually well adapted in real situations dealing with human reports.

(3) The precise qualitative DSmC and PCR5 fusion rules can be seen as special cases of Imprecise qualitative DSmC and PCR5 fusion rules as shown in our examples.

\section{Conclusion}

In this paper, we have proposed a new approach for combining imprecise qualitative beliefs based on 2-tuple distribution function linguistic representation model presented 
here, which is more general and simpler model. This approach allows the combination of information in the situations where no precise qualitative information is available. The underlying idea is to work with refined labels expressed as 2-tuples to keep working on the original set of linguistic labels. We have proposed precise and imprecise qualitative operators for 2-tuple labels and we have shown through very simple examples how we can combine precise and/or imprecise qualitative beliefs. The results obtained by this approach are more precise than those based on 1-tuple representation since no rounding approximation is done in operations and all the information is preserved in the fusion process. The imprecise qualitative DSmC and PCR5 fusion rules are the extensions of precise qualitative DSmC and PCR5 fusion rules. Applications of this approach for decision-making support in robotics are currently under development and will make the object of forthcoming publications.

Acknowledgements This work is supported by National Natural Science Foundation of China under Grant No. 60804063.

\section{References}

1. Denœux T (1999) Reasoning with imprecise belief structures. Int J Approx Reas 20:79-111 (published preliminary as Heudiasys 97/44 Technical Report)

2. Dezert J, Smarandache F (2004) Fusion of imprecise beliefs. In: Smarandache F, Dezert J (eds) Advances and applications of DSmT for information fusion (collected works), vol 1. American Research Press, Rehoboth.

3. Dezert J, Smarandache F (2008) A new probabilistic transformation of belief mass assignment. In: Proceedings of fusion 2008 international conference, Cologne, Germany, July 2008

4. Dubois D, Prade H (2001) Decision-theoretic foundations of qualitative possibility theory. Eur J Oper Res 128(3):459-478

5. Dubois D, Prade H (1993) Qualitative reasoning with imprecise probabilities. J Intell Inf Syst 2(4):319-363

6. Ferson S, Donald S (1998) Probability bounds analysis. In: Proceedings of international conference on probabilistic safety assessment and management (PSAM4). Springer, New York

7. Wang J-H, Hao JY (2006) A new version of 2-tuple fuzzy linguistic representation model for computing with words. IEEE Trans Fuzzy Syst 14(3):435-445

8. Wang J-H, Hao JY (2007) An approach to computing with words based on canonical characteristic values of linguistic labels. IEEE Trans Fuzzy Syst 15(4):593-603

9. Hájek P, Harmancová D, Verbrugge R (1995) A qualitative fuzzy possibilistic logic. Int J Approx Reas 12(1):1-19

10. Herrera F, Martínez L (2000) A 2-tuple fuzzy linguistic representation model for computing with words. IEEE Trans Fuzzy Syst 8(6):746-752

11. Herrera F, Martínez L (2001) A model based on linguistic 2tuples for dealing with multi-granular hierarchical linguistic contexts in multi-expert decision-making. IEEE Trans Syst Man Cybern 31(2):227-234

12. Herrera F, Herrera-Viedma E, Martínez L (2008) A fuzzy linguistic methodology to deal with unbalanced linguistic term sets. IEEE Trans Fuzzy Syst 16(2):354-370
13. Li X (2007) Research on fusion method of imperfect information from multi-source and its application. $\mathrm{PhD}$ thesis, Huazhong University of Science and Technology, China, June 2007

14. Li X, Huang X, Dezert J, Smarandache F (2007) Enrichment of qualitative beliefs for reasoning under uncertainty. In: Proceedings of fusion 2007 international conference, Québec, Canada, July 2007

15. Kifer M, Subrahmanian VS (1991) Theory of generalized annotated logic programs and its applications. J Log Program

16. Parsons S (1993) Qualitative methods for reasoning under uncertainty. PhD thesis, Department of Electrical Engineering, Queen Mary and Westfield College

17. Parsons S, Mamdani E (1993) Qualitative Dempster-Shafer theory. In: Proceedings of the third EMACS international workshop on qualitative reasoning and decision technologies, Barcelona, Spain

18. Parsons S (1994) Some qualitative approaches to applying Dempster-Shafer theory. Inf Decis Technol 19:321-337

19. Polya G (1954) Patterns of plausible inference. Princeton University Press, Princeton

20. Shafer G (1976) A mathematical theory of evidence. Princeton University Press, Princeton

21. Smarandache F, Dezert J (eds) (2004) Advances and applications of DSmT for information fusion (collected works), vol 1. American Research Press, Rehoboth. http:// www.gallup.unm.edu/ smarandache/DSmT-book1.pdf

22. Smarandache F, Dezert J (2005) Information fusion based on new proportional conflict redistribution rules. In: Proceedings of fusion 2005, Philadelphia, USA, July 2005

23. Smarandache F, Dezert J (eds) (2006) Advances and applications of DSmT for information fusion (collected works), vol 2. American Research Press, Rehoboth. http://www.gallup. unm.edu/ smarandache/DSmT-book2.pdf

24. Smarandache F, Dezert J (eds) (2009) Advances and applications of DSmT for information fusion (Collected works), vol 3. American Research Press, Rehoboth

25. Smarandache F, Dezert J (eds) (2007) Qualitative belief conditioning rules (QBCR). In: Proceedings of fusion 2007 international conference, Québec, Canada, July 2007

26. Smets P, Kennes R (1994) The transferable belief model. Artif Intell 66:191-243

27. Subrahmanian VS (1987) On the semantics of quantitative logic programs. In: Proceedings of the 4th IEEE symposium on logic programming

28. Walley P (1991) Statistical reasoning with imprecise probabilities. Chapman and Hall, New York

29. Wellman MP (1994) Some varieties of qualitative probability. In: Proceedings of the 5th international conference on information processing and the management of uncertainty (IPMU 1994), Paris, France, July 1994

30. Yager RR (2004) On the retranslation process in Zadeh's paradigm of computing with words. IEEE Trans Syst Man Cybern 34(2):1184-1195

31. Zadeh L (1975) Concept of a linguistic variable and its application to approximate reasoning. Inf Sci 8(1):199-249

32. Zadeh L (1979) A theory of approximate reasoning. Mach Intell 9:149-194

33. Zadeh L (1996) Fuzzy logic $=$ computing with words. IEEE Trans Fuzzy Syst 4(2):103-111

34. Zadeh L (1997) Towards a theory of fuzzy information granulation and its centrality in human reasoning and fuzzy logic. Fuzzy Sets Syst 19:111-127

35. Zadeh L (1998) Some reflections on soft computing, granular computing and their roles in the conception, design and utilization of information/intelligent systems. Soft Comput 2:23-25 